\title{
MEASUREMENT of Protein 53 Diffusion Coefficient in Live HeLa Cells Using Raster Image Correlation Spectroscopy (RICS)
}

\author{
Sungmin Hong ${ }^{1}$, Ying-Nai Wang ${ }^{2}$, Hirohito Yamaguchi ${ }^{2}$, Harinibytaraya Sreenivasappa ${ }^{1}$, \\ Chao-Kai Chou ${ }^{2}$, Pei-Hsiang Tsou ${ }^{1}$, Mien-Chie Hung ${ }^{2}$, Jun Kameoka, ${ }^{1,2}$
}

${ }^{1}$ Department of Electrical and Computer Engineering, Texas A \& M University, College Station, Texas, USA; ${ }^{2}$ Department of Molecular and Cellular Oncology, University of Texas, M. D. Anderson Cancer Center, Houston, Texas, USA.

Email:kameoka@ece.tamu.edu, mhung@mdanderson.org

Received August 24 $4^{\text {th }}, 2010$; revised September $14^{\text {th }}, 2010$; accepted October $21^{\text {st }}, 2010$.

\begin{abstract}
We have applied Raster Image Correlation Spectroscopy (RICS) technique to characterize the dynamics of protein 53 (p53) in living cells before and after the treatment with DNA damaging agents. HeLa cells expressing Green Fluorescent Protein (GFP) tagged p53 were incubated with and without DNA damaging agents, cisplatin or eptoposide, which are widely used as chemotherapeutic drugs. Then, the diffusion coefficient of GFP-p53 was determined by RICS and it was significantly reduced after the drug treatment while that of the one without drug treatment was not. It is suggested that the drugs induced the interaction of $p 53$ with either other proteins or DNA. Together, our results demonstrated that RICS is able to detect the protein dynamics which may be associated with protein-protein or protein-DNA interactions in living cells and it may be useful for the drug screening.
\end{abstract}

Keywords: Raster Image Correlation Spectroscopy, Diffusion Coefficient, p53, DNA Damage

\section{Introduction}

Analysis of protein-protein or protein-DNA interaction is indispensable for current molecular biology to understand various signaling pathways that are essential for maintenance of cellular functions in living cells. To this end, several biochemical and molecular biological techniques have been developed, such as far western blot [1], co-immunoprecipitation [2], Mass spectrometry [3], electromobility shift assay (EMSA) [4], and chromatin immunoprecipitation (ChIP) [5]. Although these techniques have their own advantages, they require long process time and a large amount of samples. More importantly, these techniques do not provide the information regarding the high spatial and temporal interaction dynamics that may provide the novel insight into current biology. Thus, several techniques, for example, Single Particle Tracking (SPT) [6], Fluorescence Recovery after Photo bleaching (FRAT) [7], and Foster Resonance Energy Transfer (FRET) [8,9], have been developed to provide higher temporal/spatial resolution for molecular dynamics in living cells. Recently, Digman and coworkers introduced the new approach, called as Raster Image
Correlation Spectroscopy (RICS), which enables to measure the protein dynamics in a living cell by using commercial laser scanning confocal microscope without adding additional expensive components. The detailed theories are described elsewhere [10-13]. Briefly, RICS can analyze the spatial fluctuation in the fluorescence signal, which is generated by the movement of fluorescence labeled molecules, to obtain molecular diffusion information.

The tumor-suppressor protein $\mathrm{p} 53$, that has been known as "the guardian of the genome," is frequently mutated or deleted in variety of human cancer types and plays an essential role in tumorigenesis [14]. In response to DNA damage, p53 is phosphorylated at several serine/threonine residues, resulting in its stabilization and activation [15]. Activated p53 forms a complex with multiple transcription co-factors and binds to promoter regions of target genes such as p21, Bax GADD45 and Puma that are involved in cell cycle arrest or apoptosis $[16,17]$. Cisplatin and etoposide are DNA damaging agents that have been used as chemotherapeutic drugs. Cisplatin unwind intra- and interstrand crosslinking of 
DNA while etoposide disrupts DNA replication and repair by inhibiting topoisomerase II enzyme [18,19]. Thus, both anti-cancer agents damage to DNA that induce p53 accumulation and activation.

In this paper, we have investigated the effects of DNA damaging agents, cisplatin and etoposide, on p53 dynamics in living HeLa cells by using RICS. After the drug treatment, the significant reductions of p 53 mobility were observed compared to the one without drug treatment. Both cisplatin and etoposide induced DNA damage that stabilized and activated p53, resulting in the formation of the DNA-p53-transcription co-factors complex. Therefore, the results obtained by RICS explain the p53 dynamics in living cells.

\section{Materials and Methods}

\subsection{Cell Culture and Plasmid Preparation, Transfection, and Drug Treatment}

Human cervical cancer, HeLa cells were obtained from American Type Culture Collection (ATCC) and maintained in DMEM/F12 medium supplemented with $10 \%$ fetal bovine serum and antibiotics. pEGFP-C2 was obtained from Clontech laboratories. p53 Open Reading Frame was digested out from pcDNA3-myc-p53 with EcoRI and XhoI, and ligated into pEGFP-C2 EcoRI/SalI sites. pcDNA3-myc-p53 was prepared by polymerase chain reaction (PCR) and described previously [20]. EGFP empty vector or GFP-p53 expression plasmid was transfected into HeLa cells using electroporation. After 24 hours transfection, the cells were seeded in $50 \mathrm{~mm}$ Glass Bottom culture dish (MatTek Corp.) at around 50 $\%$ density and cultured for additional 12 hours. Since our preliminary experiments showed that over $50 \mu \mathrm{M}$ drugs induced apoptotic cell death after 24 hours, the cell samples were treated or untreated with either $20 \mu \mathrm{M}$ cisplatin (Sigma) or $20 \mu \mathrm{M}$ etoposide (Sigma) for the different periods of time and subjected to confocal microscopy analysis.

\subsection{Confocal Microscope}

The confocal fluorescent microscopy (Olympus FV100) equipped with air-cooled $488 \mathrm{~nm}$ argon ion laser was employed for this study (Figure 1). The series of images were collected using $60 \mathrm{X}$ water immersion objective $(\mathrm{NA}=1.2)$. The scan speed was set at $12.5 \mu \mathrm{s} / \mathrm{pixel}$. The scan area was $256 \times 256$ pixels and 100 frames were collected for each sample. The corresponding line and the frame time were $4.325 \mathrm{~ms}$ and $1.150 \mathrm{~s}$, respectively. $488 \mathrm{~nm}$ wavelength of laser with $1.5 \%$ power was used for the GFP excitation, and emission spectrum was filtered between 500 and $600 \mathrm{~nm}$. The microscope was op- erated in the pseudo photon counting mode. The beam waist radius was calibrated using $10 \mathrm{nM}$ fluorescein in $0.01 \mathrm{M} \mathrm{NaOH}$ at the beginning of experiment, and it was $0.5 \mu \mathrm{m}$. The collected fluorescence data were analyzed using the Globals software package developed at the Laboratory for Fluorescence Dynamics at the University of California at Irvine [21].

\section{Results}

Figure 2 showed the RICS analysis for GFP alone in living HeLa cells immediately after adding cisplatin and etoposide. The diffusion coefficients were measured every 4 hours after adding the drugs. The GFP samples were used to calibrate the RICS analysis. The autocorrelation spectrum after background subtraction showed that GFP diffused freely into the nucleus. The measured diffusion coefficients of GFP were $38.26 \pm 5.62 \mu \mathrm{m}^{2} / \mathrm{s}$ (cistplatin-treated) and $41.32 \pm 9.81 \mu \mathrm{m}^{2} / \mathrm{s}$ (etoposidetreated) at the 0 hour. Also, consistent values were observed over time $\left(43.73 \pm 6.57 \mu \mathrm{m}^{2} / \mathrm{s}\right.$ and $44.36 \pm 6.82$ $\mu \mathrm{m}^{2} / \mathrm{s}$ at the 16 hour) as shown in Table 1 .

To compare the dynamics of $\mathrm{p} 53$ in response to DNA damaging agents, HeLa cells expressing GFP-tagged p53 were exposed to cisplatin or etoposide and subjected to RICS analysis. We first collected 100 frame images of GFP-p53 immediately after drugs treatment. Following, GFP-p53 in HeLa cells treated with the drugs were monitored every 4 hours upto 16 hours.

Figure 3 showed the auto-correlation of confocal images and fitting of the spatial correlation function at 16 hours after drugs treatment. These results suggest that GFP-p53 interacts with other molecules such as proteins and DNAs after the drug treatment and, as a result, the diffusion coefficients were reduced. As shown in Figure 4, the measured diffusion coefficients of GFP-p53 were $19.92 \pm 3.64 \mu \mathrm{m}^{2} / \mathrm{s}$ and $18.76 \pm 2.68 \mu \mathrm{m}^{2} / \mathrm{s}$ immediately after adding cisplatin and etoposide, repectively, and these results were in a good agreement with previous

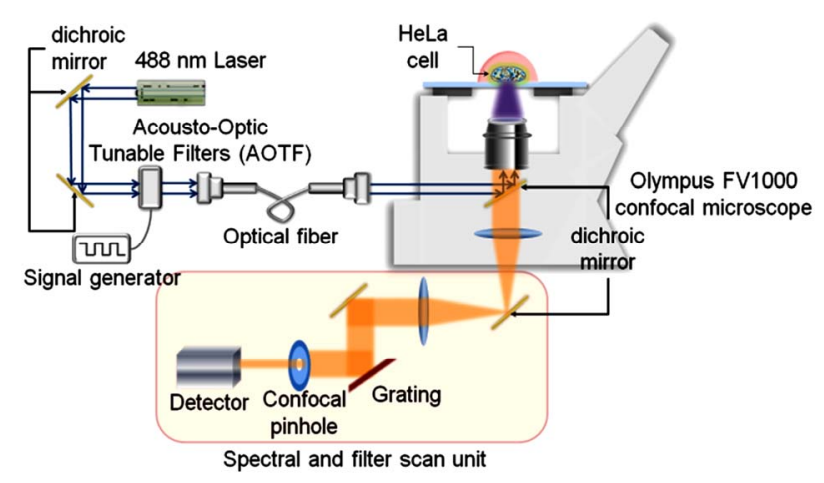

Figure 1. Schematic diagram of system setting. 
(a)
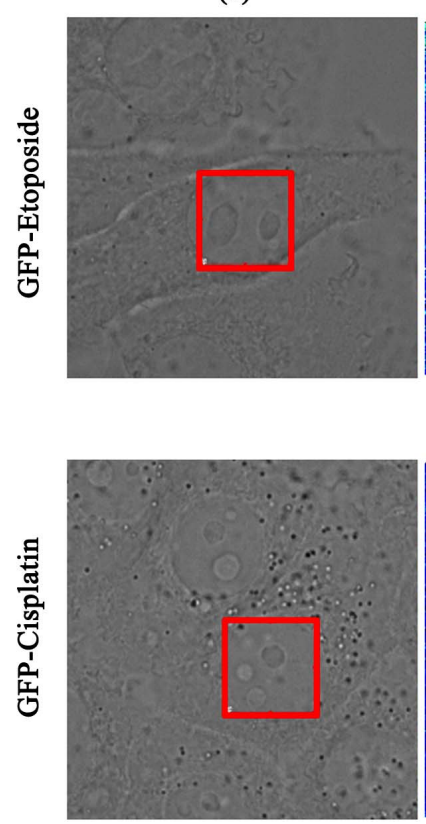

(b)
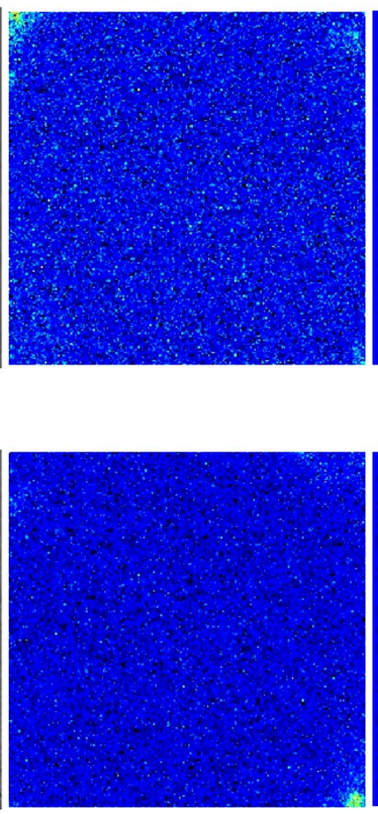

(c)
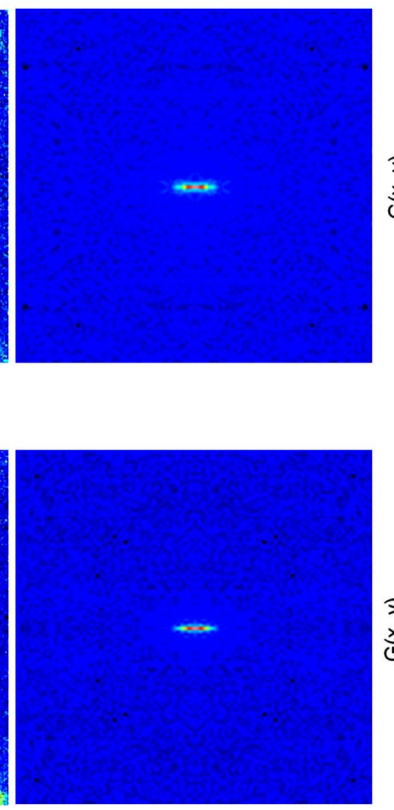

(d)

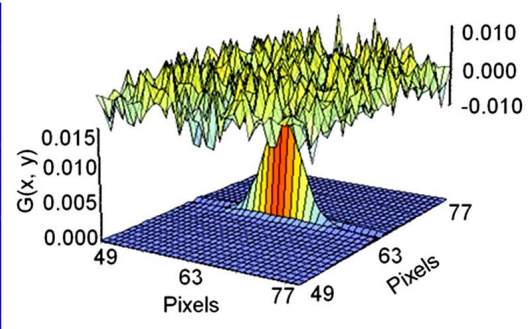

Figure 2. RICS analysis of GFP in live HeLa cells at 0 hour after anti-cancer drugs treatment. (a) Optical images of HeLa cell with the region of interest (ROI) for RICS analysis, (b) intensity images of ROI (nucleus), (c) RICS autocorrelation function of $128 \times 128$ pixels, (d) fit (lower surface) and residues (upper surface) of the spatial correlation function.

Table 1. Summary of diffusion coefficient in the nuleus of HeLa cells.

\begin{tabular}{|c|c|c|c|c|c|c|}
\hline & & \multicolumn{5}{|c|}{ Diffusion Coefficient $\left(\mu \mathrm{m}^{2} / \mathrm{s}\right)$} \\
\hline & & $0 \mathrm{hr}$ & $4 \mathrm{hr}$ & $8 \mathrm{hr}$ & $12 \mathrm{hr}$ & $16 \mathrm{hr}$ \\
\hline \multirow{2}{*}{ Cisplatin } & GFP & $38.26 \pm 5.62$ & $37.55 \pm 5.67$ & $42.65 \pm 9.46$ & $44.72 \pm 9.14$ & $43.73 \pm 6.57$ \\
\hline & GFP-p53 & $19.92 \pm 3.64$ & $8.23 \pm 5.78$ & $3.25 \pm 0.38$ & $3.21 \pm 1.18$ & $3.28 \pm 2.87$ \\
\hline Etoposide & GFP-p53 & $18.76 \pm 2.68$ & $12.77 \pm 5.42$ & $3.05 \pm 0.60$ & $3.57 \pm 1.08$ & $3.25 \pm 1.36$ \\
\hline
\end{tabular}

reported result $\left(15.4 \pm 5.6 \mu \mathrm{m}^{2} / \mathrm{s}\right)$ [22]. The diffusion dynamics of GFP-p53 were gradually decreased over time, and significant reductions of GFP-p53 mobility were observed at $8 \mathrm{hr}$ after drugs injection, $(3.25 \pm 0.38$ $\mu^{2} / \mathrm{s}$ for cisplatin and $3.05 \pm 0.60 \mu \mathrm{m}^{2} / \mathrm{s}$ for etoposide).Then, it maintained the constant values after $8 \mathrm{hr}$ in the presence of both drugs. The diffusion dynamics changes of GFP-p53 in response to the drugs were summarized in Table $\mathbf{1}$.

\section{Discussion}

In this work, we measured the diffusion coefficient of GFP-tagged p53 in the nucleus of HeLa cells using RICS approach. Also, DNA damaging agents were used to verify p53 dynamics in response to DNA damage.
The diffusion coefficients of GFP obtained by RICS were agreed well with previously reported value. Hinow et al. applied free diffusion model to explain the mobility of GFP in the nucleus of H1299 human large cell lung carcinoma cell using confocal FRAP technique, and they reported the diffusion coefficient of GFP $(41.6 \pm 13.6$ $\mu \mathrm{m}^{2} / \mathrm{s}$ ) [22]. Since GFP was not involved in DNA damage response, the diffusion of GFP was not affected by anti-cancer drugs. This demonstrated that RICS method could provide the stable result in measuring diffusion coefficient in living HeLa cells.

After DNA damaging agent treatment, the significant reductions in GFP-p53 mobility were observed in the nucleus. It is well known that p53 is stabilized and activated in response to DNA damage [23]. In this study, 
(a)

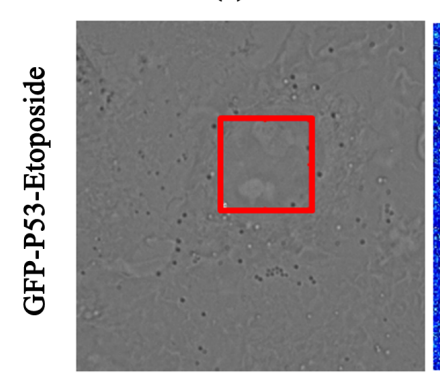

(b)
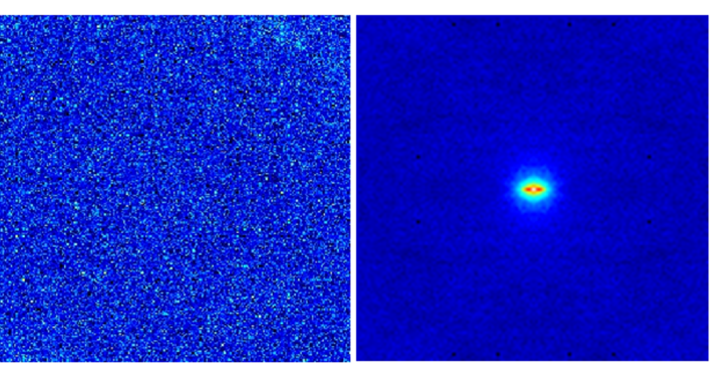

(c)
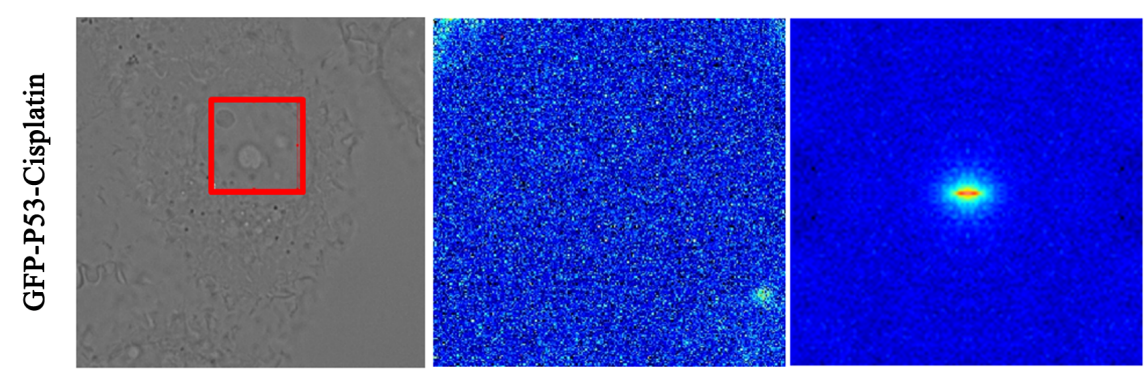

(d)

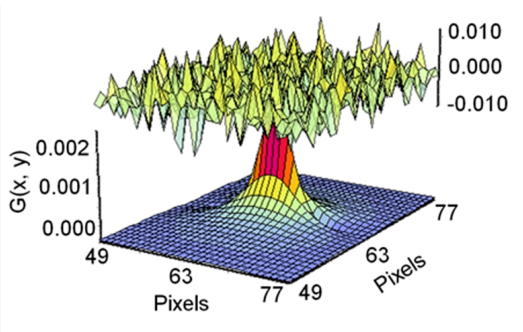

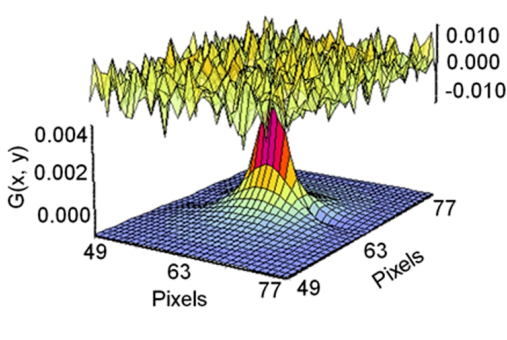

Figure 3. RICS analysis of GFP-p53 in live HeLa cells at 16 hours after anti-cancer drugs treatment. (a) optical images of HeLa cell with the region of interest (ROI) for RICS analysis, (b) intensity images of ROI (nucleus), (c) RICS autocorrelation function of $128 \times 128$ pixels, (d) fit (lower surface) and residues (upper surface) of the spatial correlation function.

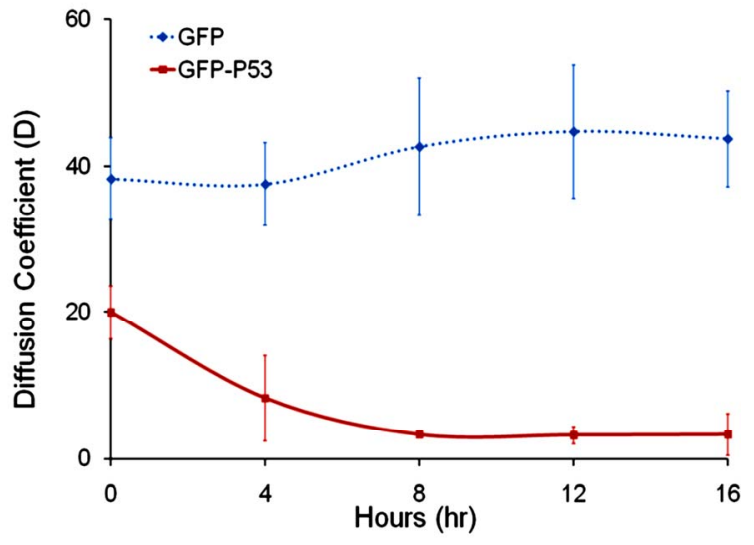

(a)

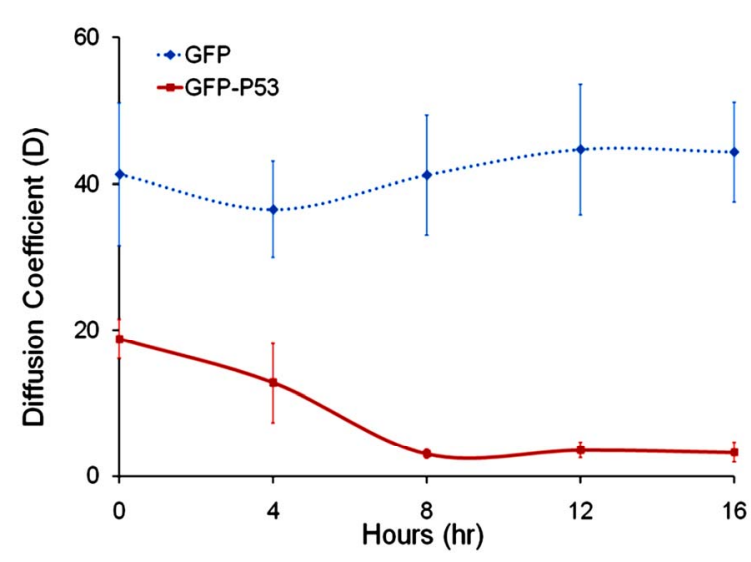

(b)

Figure 4. The diffusion coefficient graph as a function of time. (a) diffusion dynamics changes of GFP or GFP-p53 in cisplatin treated cells, (b) diffusion dynamics changes of GFP or GFP-p53 in etoposide treated cells.

$20 \mu \mathrm{M}$ concentrations of cisplatin and etoposide were used. It would be expected that higher concentration of drugs induces the quicker reduction of the mobility of GFP-p53 due to the p53 activation by more DNA damage. Furthermore, it has been known that ciplatin induces single stranded break of DNA [24] while etopside causes double stranded break of DNA [25]. Thus, the combination of these drugs induces more DNA damage, and it also would be expected that the combination of both drugs induces the quicker reduction of the mobility of

\section{GFP-p53.}

Moreover, it has been shown that p53 translocates from cytosol to the nucleus after DNA damage. Activated p53 form a complex with multiple transcriptions co-factors and binds to the specific promoter region in DNA to induce target genes within 8 hours. As a result, p53 mobility was expected to be decreased. Our results for fluctuations of GFP-p53 diffusion coefficients were consistent with our prediction from the current knowledge regarding p53. 


\section{Conclusions}

In conclusion, we have successfully measured the diffusion coefficients of GFP-p53 in living HeLa cells subjected to DNA damage agents by using commercial confocal microscope to RICS analysis method. RICS is able to measure protein diffusion in live cells using regular confocal microscope and require relatively short period of time. Therefore, it may be applied to a large-scale, high throughput drug screening based on the activation or inactivation of tumor suppressors or oncogene products in the future.

\section{Acknowledgements}

This project has been supported by the CMUH Cancer Research Center of Excellence DOH 99-7D-C-111-005, Taiwan, Institute of Basic Science, China Medical University and Hospital/M. D. Anderson Cancer Center Sister Institution Fund, CCSG CA16672, NIH R 01 CA109311, NIH PO1 099031, and US Army Department Breast Cancer Research Program W81XWH-08-1-064901 to M.-C.H.; NIH R21 CA135318-01A1 and US Army Department Breast Cancer Research Program W81XWH-08-1-0644 to J.K. The use of the Micrsoscopy and Imaging Center facility at Texas A \& M University is acknowledged. The Olympus FV1000 confocal microscope acquisition was supported by the Office of the Vice President for Research at Texas A \& M University.

\section{REFERENCES}

[1] Y. Wu, Q. Li and X.-Z. Chen, "Detecting Protein-Protein Interactions by Far Western Blotting," Nature Protocols, Vol. 2, No. 12, 2007, pp. 3278-3284.

[2] P. Yaciuk, "Co-Immunoprecipitation of Protein Com-plexes," Methods in Molecular Medicine, Vol. 131, 2007, pp. 103-111.

[3] D. Figeys, L. D. McBroom and M. F. Moran, "Mass Spectrometry for the Study of Protein-Protein Interac-tions," Methods, Vol. 24, No. 3, 2001, pp. 230-239.

[4] M. Gaudreault, M.-E. Gingras, M. Lessard, S. Leclerc and S. L. Guérin, "Electrophoretic Mobility Shift Assays for the Analysis of DNA-Protein Interactions," Methods in Molecular Biology, Vol. 543, 2009, pp. 15-35.

[5] B. G. Hoffman and S. J. M. Jones, "Genome-Wide Identification of DNA-protein Interactions Using Chromatin Immunoprecipitation Coupled with Flow Cell Sequencing," Journal of Endocrinol, Vol. 201, No. 1, 2009, pp. $1-13$.

[6] K. Jaqaman, D. Loerke, M. Mettlen, H. Kuwata, S. Grin-stein, S. L. Schmid, et al., "Robust Single-Particle Tracking in Live-cell Time-lapse Sequences," Nature Methods, Vol. 5, No. 8, 2008, 695-702.
[7] D. Axelrod, D. E. Koppel, J. Schlessinger, E. Elson and W. W. Webb, "Mobility Measurement by Analysis of Fluorescence Photobleaching Recovery Kinetics," Biophysical Journal, Vol. 16, No. 9, 1976, pp. 1055-1069.

[8] P. Roy, Z. Rajfur, P. Pomorski and K. Jacobson, "Microscope-based Techniques to Study Cell Adhesion and Migration," Nature Cell Biology, Vol. 4, No. 4, 2002, pp. E91-E96.

[9] I. A. Demarco, A. Periasamy, C. F. Booker and R. N. Day, "Monitoring Dynamic Protein Interactions with Photoquenching FRET," Nature Methods, Vol. 3, No. 7, 2006, pp. 519-524.

[10] M. A. Digman, P. Sengupta, P. W. Wiseman, C. M. Brown, A. R. Horwitz and E. Gratton, "Fluctuation Correlation Spectroscopy with a Laser-Scanning Microscope: Exploiting the Hidden Time Structure," Biophysical Journal, Vol. 88, No. 5, 2005, pp. L33-L36.

[11] M. A. Digman, C. M. Brown, P. Sengupta, P. W. Wise-man, A. R. Horwitz and E. Gratton, "Measuring Fast Dynamics in Solutions and Cells with a Laser Scanning Microscope," Biophysical Journal, Vol. 89, No. 2, 2005, pp, 1317-1327.

[12] C. M. Brown, R. B. Dalal, B. Hebert, M. A. Digman, A. R. Horwitz and E. Gratton, "Raster Image Correlation Spectroscopy (RICS) for Measuring Fast Protein Dynamics and Concentrations with a Commercial Laser Scanning Confocal Microscope," Journal of Microscopy, Vol. 229, No. 1, 2008, pp. 78-91.

[13] M. A. Digman and E. Gratton, "Analysis of Diffusion and Binding in Cells Using the RICS Approach," Microscopy Research and Technique, Vol. 72, No. 4, 2009, pp. 323332.

[14] [14] K. M. Ryan, A. C. Phillips and K. H. Vousden, "Regulation and Function of the p53 Tumor Suppressor Protein," Current Opinion in Cell Biology, Vol. 13, No. 3, 2001, pp. 332-337.

[15] A. M. Bode and Z. Dong, "Post-Translational Modification of p53 in Tumorigenesis," Nature Reviews Cancer, Vol. 4, No. 10, 2004, pp. 793-805.

[16] O. Laptenko and C. Prives, "Transcriptional Regulation by p53: One Protein, Many Possibilities," Cell Death \& Differentiation, Vol. 13, No. 6, 2006, pp. 951-961.

[17] J. K. Sax and W. S. El-Deiry, "p53 Downstream Targets and Chemosensitivity," Cell Death \& Differentiation, Vol. 10, No. 4, pp. 413-417.

[18] M. Jiang, X. Yi, S. Hsu, C.-Y. Wang and Z. Dong, "Role of p53 in Cisplatin-induced Tubular Cell Apoptosis: Dependence on p53 Transcriptional Activity," American Journal of Physiology Renal Physiology, Vol. 287, No. 6, 2004, pp. F1140-1147.

[19] P. J. Smith, (1990), "DNA Topoisomerase Dysfunction: A New Goal for Antitumor Chemotherapy," BioEssays, Vol. 12, No. 4, pp. 167-172.

[20] H. Yamaguchi, N. T. Woods, L. G. Piluso, H.-H. Lee, J. Chen, K. N. Bhalla, et al., "p53 Acetylation Is Crucial for Its Transcription-Independent Proapoptotic Functions," 
Journal of Biological Chemistry, Vol. 284, No. 17, 2009, 11171-11183.

[21] J. Beechem, E. Gratton, M. Ameloot, J. Knutson and L. Brand, "The Global Analysis of Fluorescence Intensity and Anisotropy Decay Data: Second-Generation Theory and Programs," Topics in Fluorescence Spectroscopy, Vol. 2, 2002, pp. 241-305.

[22] P. Hinow, C. E. Rogers, C. E. Barbieri, J. A. Pietenpol, A. K. Kenworthy and E. DiBenedetto, "The DNA Binding Activity of p53 Displays Reaction-Diffusion Kinetics," Biophysical Journal, Vol. 91, No. 1, 2006, pp. 330-342.

[23] D. P. Lane, "p53, Guardian of the Genome," Nature, Vol.
358, No. 6381, 1992, pp. 15-16.

[24] L. Yen, N. Zeng-Rong, X.-L. You, S. Richard, B. C. Langton-Webster and M. A. Alaoui-Jamali, "Regulation of Cellular Response to Cisplatin-induced DNA Damage and DNA Repair in Cells Overexpressing p185erbB-2 is Dependent on the Ras Signaling pathway," Oncogene, Vol. 14, No. 15, 1997, pp. 1827-1835.

[25] S. A. S. Walles, R. Zhou and E. Liliemark, "DNA Damage Induced by Etoposide; A Comparison of Two Different Methods for Determination of Strand Breaks in DNA," Cancer Letters, Vol. 105, No. 2, 1996, pp. 153159. 